\title{
INTEgRaCión de SABERES EN LA PARROQUia PEDRO PABLO GóMEZ DEL CANTÓN JIPIJAPA
}

AUTORES: $\quad$ Raquel Vera Velázquez. ${ }^{1}$

Kirenia Maldonado Zúñiga ${ }^{2}$

Wilfrido Javier Del Valle Holguín ${ }^{3}$

Pedro Roberto Valdés Tamayo ${ }^{4}$

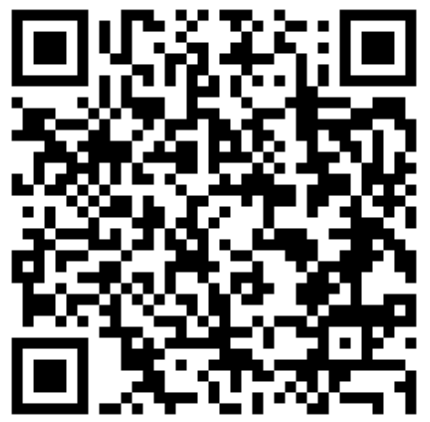

DIRECCIÓN PARA CORRESPONDENCIA: vera-raquel@unesum.edu.ec.

Fecha de recepción: 25/01/2020

Fecha de aceptación: 22/02/2020

\section{RESUMEN}

El suelo es un recurso finito, y los procesos naturales para su formación, desarrollo y restauración no ocurren a la misma velocidad a la que se degrada. En el presente artículo se definen y describen las principales propiedades físicas, químicas y biológicas del suelo, discutiendo su importancia y los valores típicos de los suelos agrícolas. Se hace una reflexión sobre la importancia de monitorear las propiedades del suelo y su manejo dirigido para evitar su degradación. Se analizan las relaciones interdisciplinarias entre las ciencias básicas para realizar el estudio del suelo agrícola de la parroquia rural la Pedro Pablo Gómez del cantón Jipijapa. Los métodos utilizados fueron exploratorio documental, a través del estudio e investigación bibliográfica, estadístico con la documentación de la parroquia y la experiencia de los pobladores de la parroquia. La gran presión sobre los suelos que la humanidad ejerce para satisfacer sus necesidades de alimento ha promovido su uso intensivo que, junto con las malas prácticas de manejo (tales como la labranza intensiva, monocultivo, aplicación indiscriminada de agroquímicos, entre otros), han provocado deterioro y degradación edáfica. El problema de fondo es el escaso entendimiento del ser humano sobre el suelo y sus propiedades por estas razones nos dimos a la tarea de investigar mediante los proyectos integradores de saberes las situaciones del suelo agrícola de las parroquias de la zona 4 provincia de Manabí para ayudar a la comunidad de Jipijapa en la capacitación y estudio del suelo para tener un mejor resultado en sus cosechas.

PALABRAS CLAVE: Ciencias básicas, suelo agrícola, métodos estadísticos, integración, saber

INTEGRATION OF KNOWLEDGE IN THE PEDRO PABLO GÓMEZ DEL CANTÓN JIPIJAPA PARISH

\footnotetext{
${ }^{1}$ Universidad Estatal del Sur de Manabí, Facultad de Ciencias Naturales y de la Agricultura, Jipijapa, Ecuador

2 Universidad Estatal del Sur de Manabí, Facultad de Sistemas Computacionales, Jipijapa, Ecuador.

${ }^{3}$ Universidad Estatal del Sur de Manabí, Facultad de Ciencias Naturales y de la Agricultura, Jipijapa, Ecuador.

${ }^{4}$ Universidad Estatal del Sur de Manabí, Facultad de Ciencias Naturales y de la Agricultura, Jipijapa, Ecuador.
} 
Raquel Vera Velázquez., Kirenia Maldonado Zúñiga, Wilfrido Javier Del Valle Holguín...

\section{ABSTRACT}

Soil is a finite resource, and the natural processes for its formation, development, and restoration do not occur at the same rate at which it degrades. In this article the main physical, chemical and biological properties of the soil are defined and described, discussing its importance and the typical values of agricultural soils. A reflection is made on the importance of monitoring the properties of the soil and its directed management to avoid its degradation. The interdisciplinary relationships between the basic sciences are analyzed to carry out the study of agricultural land in the rural parish of Pedro Pablo Gómez in the canton of Jipijapa. The methods used were exploratory documentary, through bibliographic study and research, statistics with the documentation of the parish and the experience of the residents of the parish. The great pressure on the soils that humanity exerts to satisfy their food needs has promoted their intensive use which, together with poor management practices (such as intensive tillage, monoculture, indiscriminate application of agrochemicals, among others), have caused deterioration and soil degradation. The underlying problem is the poor understanding of the human being about the soil and its properties. For these reasons, we undertook the task of investigating the situations of agricultural soil in the parishes of zone 4, Manabí province, through the integrative projects of knowledge. to the Jipijapa community in the training and study of the soil to have a better result in their harvests.

KEYWORDS: Basic sciences, agricultural soil, statistical methods, integration, knowing

\section{INTRODUCCIÓN}

El modelo de producción agropecuaria que en la zona sur de Manabí ha imperado por muchos años para desarrollar la agricultura se concibió bajo el paradigma de los rendimientos de los cultivos, esto ha traído consigo la manifestación de graves problemas de plagas, así como la aparición de procesos de degradación de suelos como compactación y erosión, además de otros efectos colaterales por contaminación de suelos, aguas subterráneas, ríos, perdidas en la biodiversidad, no solamente en los sistemas agrícolas, sino también en los ecosistemas naturales.

El Proyecto Integrador de Saberes que se desarrollará en el primer nivel de la carrera Agropecuaria se relaciona con problemas de la realidad, identificados en las labores previas de vinculación con la sociedad, practicas pre-profesionales y en los estudios de documentos normativos.

Este proyecto, así como todos los proyectos de la carrera Agropecuaria, tienen relación con los problemas de las ciencias y de la zona 4, los cuales directamente utilizan los conocimientos de las ciencias básicas (Lógica matemática, Algebra, Física, Química general, Biología celular y Expresión oral y escrita).

Los estudiantes llegan con conocimientos diferentes en los campos del saber, algunos con muchas debilidades y criterios equivocados sobre la carrera agropecuaria, como el hecho de pensar que esta carrera no tiene relación con las ciencias básicas como la química, física o matemáticas. Muchos tienen deficiencias serías en los conocimientos básicos de estas asignaturas. Los nuevos estudiantes no ven la relación de las ciencias exactas y las ciencias biológicas, menos comprenden sobre la importancia de la interpretación y presentación de resultados.

A través de ésta investigación se pretende fortalecer los conocimientos para lograr un cambio sistémico (de actitud) de las percepciones y criterios de conocimiento, a través de lograr el desarrollo de un proyecto integrador de saberes, que articule los diferentes campos del saber 
impartidos de una manera natural y según como acontece realmente en la producción de los sistemas agropecuarios.

Sin embargo, el concepto de agricultura sostenible considera a una Unidad de Producción Agropecuaria productiva la que es capaz de producir la mayor cantidad y diversidad de los recursos necesarios, que incluye alimentos, combustible, fibra, plantas medicinales y otros. Intriago, B. R. (Diciembre de 2013). La conversión de la agricultura hacia la producción agraria sostenible implica un proceso de mejora continua de los sistemas agrícolas, que puedan satisfacer las demandas de la población en armonía con la conservación del medio ambiente. A pesar de la importancia para la vida, el suelo no ha recibido de la sociedad la atención que merece. Su degradación es una seria amenaza para el futuro de la humanidad. Por lo tanto, los científicos se enfrentan al triple desafío de intensificar, preservar e incrementar la calidad de la tierra. Para ello, es necesario contar con una sólida concepción de la calidad y con indicadores de calidad o salud de la tierra y de manejo sostenible de la misma, tal como se cuenta para dar seguimiento a variables sociales y económicas. Bautista, C. A. (2004).

Objetivo general: investigar las propiedades químicas de los suelos de la parroquia Pedro Pablo Gómez.

El suelo es un sistema dinámico que consta de tres fases (sólida, líquida y gaseosa) cuyas propiedades físicas, químicas y biológicas interactúan entre sí para mantener un equilibrio adecuado para el desarrollo de las plantas y su entorno (Tamhane et al. 1986).

Las propiedades físicas son aquellas que pueden observarse y/o medirse sin alterar químicamente la composición del suelo y están relacionadas con el movimiento del aire, calor, agua, raíces y nutrientes; entre ellas se encuentran la profundidad, textura, estructura, densidad aparente, densidad real, porosidad, color y temperatura. Parker, (2010).

Las propiedades químicas son aquellas que pueden observarse y/o medirse a partir de cambios químicos que ocurren en el suelo. Estas propiedades describen el comportamiento de los elementos, sustancias y componentes que integran el suelo (Tamhane et al. 1986); entre ellas están el pH, Capacidad de Intercambio Catiónico (CIC), conductividad eléctrica (CE), el contenido de materia orgánica (MO) y de elementos (nutritivos o tóxicos).

Las propiedades biológicas del suelo están relacionadas con la materia orgánica y con los organismos que viven en él, como las raíces de las plantas, lombrices, insectos, nematodos, hongos, bacterias, etc. (Hall 2008, FAO 2015) Las actividades de estos organismos están relacionados con el movimiento de agua y minerales, y son fundamentales en la descomposición de la MO, en el ciclo de los nutrientes, en la síntesis de sustancias húmicas y en la fijación de $\mathrm{N}$ (Tamhane et al. 1986).

El análisis del estado físico del suelo puede indicar que existen problemas en el mismo, que estarán directamente relacionados al estado químico y biológico; si se compara con un buen médico que utiliza la medicina preventiva ante síntomas visibles de sus pacientes, los productores en sus fincas como buenos médicos del suelo, podrán realizar un análisis físico con métodos sencillos y determinar el estado del suelo en que trabajan, disminuyendo los costos que traen los análisis químicos de laboratorio.

Los suelos generalmente han permanecido en una constante sobre explotación, primando el monocultivo y todo un coctel de productos tóxicos que por años han ido transformando su estado físico, químico y biológico, sin embargo actualmente, la academia a través de sus proyectos de 
Raquel Vera Velázquez., Kirenia Maldonado Zúñiga, Wilfrido Javier Del Valle Holguín...

investigación y la vinculación con la comunidad está impulsando prácticas más amigables con el medioambiente, éste análisis entre suelos similares pero con distintas prácticas de manejo nos dará algunas directrices para determinar el estado del suelo desde la desde la óptica de la sostenibilidad y la agroecología.

El desconocimiento e información obsoleta de las realidades productivas de un sector para la toma de decisiones y formulación de la política pública, ha generado una problemática en su distribución de acuerdo al uso del suelo en el país, lo que ha conllevado una sobre explotación en suelos fértiles o la migración de esta actividad a sectores de áreas con fertilidad baja, susceptibles a la erosión.

Es importante destacar que un buen levantamiento de suelos como insumo, es útil para la planificación territorial y zonificación agraria. El no tener un buen levantamiento de suelos repercutirá en una planificación deficiente lo que conllevaría problemas a futuro para el territorio, lo que se traduce en subutilización y sobreutilización de las tierras,

malas prácticas agrícolas, mala ubicación de la población en lugares no aptos para ser habitados, inundaciones, entre otros.

¿Cuáles son las propiedades químicas de los suelos en la parroquia Pedro Pablo Gómez?

\section{DESARROLLO}

\section{MATERIALES Y MÉTODOS}

Talento Humano: estudiantes, tutor del Proyecto Integrador de Saberes, agricultores encuestados de la parroquia rural La América, presidente del Gobierno Autónomo Descentralizado Parroquial de La América

Materiales: computadora, pendrive, cd y útiles de oficina

Métodos: se utilizaron métodos y técnicas apropiadas para recoger y analizar la información. Descriptivo: La investigación realizada se pudo considerar descriptiva, porque tiene como propósito la descripción de un evento o fenómeno en este caso las propiedades químicas de los suelos con fines agrícolas del sector objeto de estudio, así como la frecuencia en el uso de agroquímicos y fertilizantes por parte de los agricultores del sector.

Bibliográfico: La investigación también se orientó, en un diseño documental o bibliográfico, en este sentido el proyecto recurrió a diversas fuentes de investigación sobre el tema; a libros, normas vigentes, documentos digitales los cuales optaron conceptos y teorías referentes a cada una de las variables del proyecto. La investigación de campo se empleó para diagnosticar deficiencias en los procesos de manejo y control de los activos fijos, a efectos de aplicar alternativas. Esta investigación proporciona información exacta con un alto grado de confiabilidad y por consecuencia un bajo margen de error.

Este Proyecto Integrador de Saberes se desarrolló en la parroquia rural Pedro Pablo Gómez perteneciente al cantón Jipijapa, provincia de Manabí, la misma el 24 de octubre de 1917 fue elevada a categoría de parroquia rural con el nombre de Pedro Pablo Gómez en memoria de un distinguido ciudadano Jipijapense.

Limites norte: Cantón Jipijapa sur: Manglar alto provincia de Santa Elena este: Cantón Paján oeste: Parque Nacional Machalilla. 
En la Parroquia Rural de Pedro Pablo Gómez, según el Censo de Población y Vivienda del INEC 2010, cuenta con 3.564 habitantes, de los cuales 1.897 son hombres que representa el $53 \%$ y 1.667 mujeres con el $47 \%$. Tabla \# 1

\begin{tabular}{|l|l|l|l|}
\hline PARROQUIA & $\begin{array}{l}\text { POBLACION } \\
\text { HOMBRE }\end{array}$ & $\begin{array}{l}\text { POBLACION } \\
\text { MUJER }\end{array}$ & TOTAL \\
\hline Jipijapa & 19611 & 20621 & 40232 \\
\hline $\begin{array}{l}\text { Pedro Pablo } \\
\text { Gómez }\end{array}$ & 1897 & 1667 & 3564 \\
\hline
\end{tabular}

Fuente: IGM-MAGAP-SNI. (Diciembre de 2015)

Población Pedro Pablo Gómez .Gráfico \#1

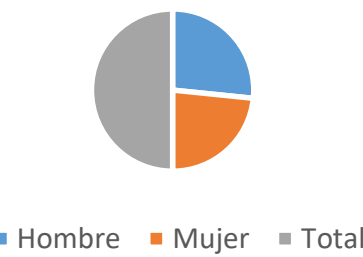

CONTEXTO TERRITORIAL. Tabla \#2.

\begin{tabular}{|l|l|}
\hline Provincia & Manabí \\
\hline Cantón & Jipijapa \\
\hline Nombre de la parroquia & Pedro Pablo Gómez \\
\hline Fecha de creación & 24 de octubre de 1917 \\
\hline Población total & 3.564 \\
\hline Extensión & $18.893,70 \mathrm{Km} 2$ \\
\hline Límites & $\begin{array}{l}\text { Norte: Cantón Jipijapa, Sur: Manglar alto provincia } \\
\text { de Santa Elena } \\
\text { Este: Cantón Paján, Oeste: Parque Nacional } \\
\text { Machalilla }\end{array}$ \\
\hline
\end{tabular}

Fuente: IGM-MAGAP-SNI. (Diciembre de 2015)

\section{RESULTADOS Y DISCUSIÓN}

El Ecuador al igual que todos los países en vías de desarrollo no ha escapado al problema del manejo inadecuado de sus tierras, por la falta de planificación y ordenamiento territorial, generando un sobre o sub utilización de las tierras. A lo largo del tiempo ha existido un abuso de los recursos naturales que afecta al sector agropecuario, tanto desde el ámbito ecológico ambiental como desde el económico y social (MAGAP, 2015).

Según estudios realizados por el Ministerio de Agricultura y Ganadería la erosión es uno de los problemas más importantes que afecta al sector agropecuario en un alto porcentaje, se produce y 
Raquel Vera Velázquez., Kirenia Maldonado Zúñiga, Wilfrido Javier Del Valle Holguín...

se incrementa por la actividad humana, y los efectos de este proceso generalmente provocan la pérdida parcial o total irreversible de los ecosistemas naturales.

Con estos antecedentes se hace necesario implementar estudios que permitan planificar el uso racional de las tierras a fin de tener explotaciones agropecuarias eficientes y reduciendo pérdida de este importante recurso. Por las razones expuestas el realizar el presente Proyecto Integrador de Saberes en la parroquia Pedro Pablo Gómez permitirá su planificación técnica y sustentable en el tiempo; este estudio también servirá de base para la planificación y ordenamiento territorial, el cual permitirá generar proyectos que sean apropiados para la zona, y el aprovechamiento de los recursos naturales.

\section{Factores Climáticos}

Según Bautista, C. A. (2004). La parroquia Pedro Pablo Gómez, cuenta con un clima tropical Sabana de Costa; existen dos estaciones invierno y verano. La temperatura media anual va desde 18 a $24{ }^{\circ} \mathrm{C}$; Promedio anual de precipitaciones de $500 \mathrm{~mm}$ a $1000 \mathrm{~mm}$ Zona Sub Humedad.

Cuenta con un clima Tropical Sabana de Costa; existen dos estaciones invierno y verano. La temperatura media anual va desde 18 a $24{ }^{\circ} \mathrm{C}$; Precipitaciones de $500 \mathrm{~mm}$ a $1000 \mathrm{~mm}$ Zona Sub Humedad. La humedad relativa se encuentra en los 78,24 como promedio anual.

La superficie de la tierra, sus variaciones caracterizadas por la altura y forma del terreno constituyen el relieve terrestre, en el cantón existe un macizo montañoso aislado e irregular, que se desarrolla entre Jipijapa y Manta, rodeado al norte y oeste por el Océano Pacifico, al sur por el valle de Jipijapa y al este por el río Portoviejo.

En este valle termina la cordillera de Colonche y las montañas costaneras que siguen hacia Bahía de Caráquez no se presentan cadenas largas, más bien son grupos macizos irregulares. El relieve del cantón está condicionado por alturas que varían desde $50 \mathrm{~m}$ hasta $800 \mathrm{msnm}$, es bastante irregular con altitudes más pronunciadas en la parte sur-este donde sobresale un cerro sin nombre con $820 \mathrm{msnm}$; en la parte centro-sur sobresale un accidente orográfico con una altitud de 635 metros.

Es necesario indicar que del total de la superficie que son 146741.60has. el 31,90\% corresponde a relieve montañoso con pendientes mayores a $70 \%$ y de acuerdo a la capacidad de uso de las tierras estas tienen severas limitaciones, independiente de sus limitaciones solas o combinadas no presentan condiciones para su utilización en actividades agrícolas o pecuarias. Bautista, C. A. (2004)

Es decir que el relieve es abrupto para la localización de ciertas actividades socioeconómicas; el relieve montañoso y la fragilidad del terreno en ciertas zonas impiden el desarrollo de actividades como la agricultura intensiva, los asentamientos humanos e infraestructuras. La localización de actividades en áreas poco apropiadas; desde el punto de vista del relieve, genera una preocupante vulnerabilidad ambiental por problemas y conflictos de uso (viviendas, agricultura de subsistencia), en áreas bajo amenaza.

En la parroquia Pedro Pablo Gómez el territorio es montañoso, los cerros atraviesan desde el sur hasta el norte, siendo estos la prolongación oriental de las montañas de Colonche.

\section{Utilización del Suelo}


De acuerdo a los datos proporcionados por el Ministerio de Agricultura Ganadería y Pesca, en la Parroquia Pedro Pablo Gómez, 38.75ha se utiliza para la protección y producción, 1337,18ha establecidas para el sector agrícola, 17983.71 ha de suelo en la actualidad se encuentran destinadas para la conservación y protección, 154.49ha para la conservación y producción, un 15.95ha es destinada para la conservación y pecuario así mismo 8639.40ha de suelo están destinadas para el sector pecuario, estas son las formas de uso de suelo en la parroquia de mayor proporción. Bautista, C. A. (2004).Tabla \# 3.

\section{USO DEL}

\section{SUELO}

\begin{tabular}{|l|l|}
\hline USO & ÁREA (ha) \\
\hline Protección o producción & 38.75 \\
\hline Agrícola & 1337.18 \\
\hline Conservación y proteccion & 17983.71 \\
\hline Conservación y producción & 154.49 \\
\hline Conservación y Pecuario & 15.95 \\
\hline Pecuario & 8639.4 \\
\hline Agropecuario mixto & 94.43 \\
\hline Antrópico & 13.38 \\
\hline
\end{tabular}

Fuente: IGM-MAGAP-SNI

Utilización del suelo gráfico \# 2

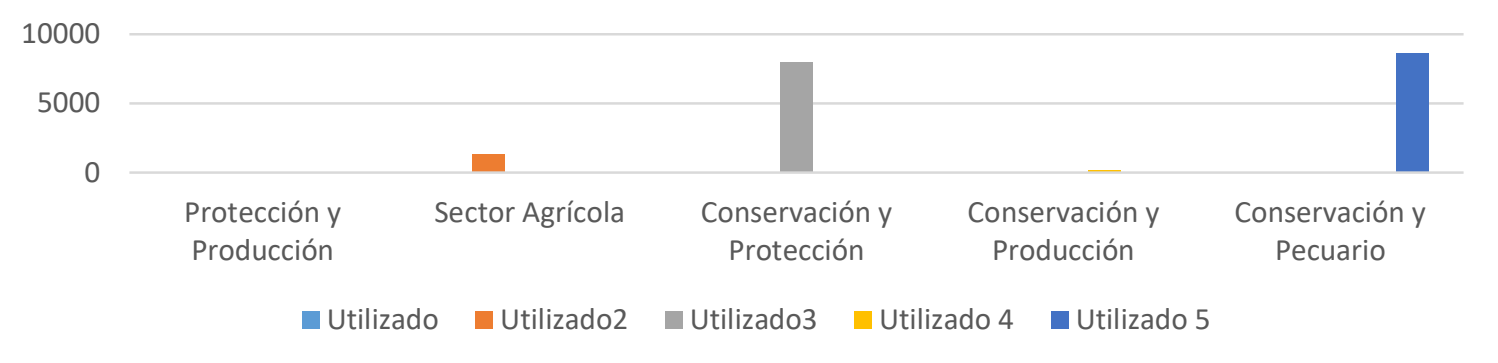

\section{Suelos con Pendientes}

La Parroquia rural de Pedro Pablo Gómez, presenta suelos con factores limitantes, textura arcillosa a arenosa, se encuentran en áreas planas o poco onduladas, profundos, de textura franco arcillosa. Tienen una estructura masiva y son sensibles a la desecación en caso de riego. Suelos con factores limitantes, pendientes de 25 a 50\%, piedras, texturas, son suelos de relieves, en su mayoría medianamente profundos, la textura puede ser muy arcillosa, con grietas abiertas más de 90 días Suelos con factores limitantes, pendientes de 50 a 70\%, profundidad débil, éstos son suelos de declives de colinas, pendientes muy fuertes, profundidad variable, pero buena textura; zonas de relieves para pastos o cultivos arbustivos en las zonas Húmedas. Gómez, G. P. (2015).Tabla \#4

\begin{tabular}{|l|l|}
\hline PENDIENTES DE PEDRO PABLO GÓMEZ & AREA HAS \\
\hline DESCRIPCIÓN & 14170.91 \\
\hline Abruptas, montañoso mayor al 70\% & 376.47 \\
\hline Débil, plano o casi plano 0-5\% & 8518.18 \\
\hline Fuertes, colinado 25-50\% & 1012.61 \\
\hline Inclinación regular, suave o ligeramente ondulada 5-12\% & 2470.63 \\
\hline Irregular, ondulacion moderada 12-25\% & 1813.13 \\
\hline Muy fuertes, escarpado 50-70\% & \\
\hline
\end{tabular}

Fuente: IGM-MAGAP-SNI. (Diciembre de 2015). 
Raquel Vera Velázquez., Kirenia Maldonado Zúñiga, Wilfrido Javier Del Valle Holguín...

Areas. has.Gráfico \# 3
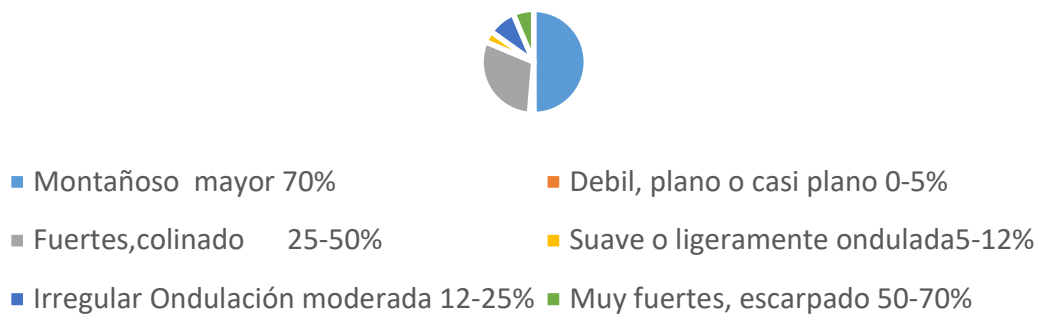

\section{Cobertura y Uso del Suelo}

Pedro Pablo Gómez, se encuentra en una cadena montañosa, con su principal característica los suelos alcalinos pesados que se agrietan en época seca y tienen origen sedimentario terciario. La topografía es generalmente irregular, utilizados generalmente para potreros, pero con ciertos sectores de topografía plana que se son utilizados para cultivos intensivos. A pesar de que se afecta la producción por las vías en mal estado y la escasez de agua en la parroquia no disminuye la producción y comercialización agropecuaria.

El 90\% de la población económicamente activa de la parroquia se dedica a la agricultura. La importancia de este indicador radica en que nos define el rumbo que se debe tomar en la formulación de propuestas para el fortalecimiento de la economía parroquial, ya que lo que se implemente en favor del sector productivo agrícola tendrá un impacto directo en la mayor parte de la población, mientras que otras actividades que se quieran implementar, si bien ampliarán la base de generación de empleo en la parroquia, su impacto será a mediano y largo plazo mas no inmediato. Bautista, C. A. (2004)

Los ingresos medios mensuales son obtenidos de la actividad que realiza el hombre y la mujer en la agricultura, ganadería y silvicultura, además obtienen la caza y la pesca para el auto consumo.

La economía parroquial tiene como eje fundamental la producción agropecuaria para la destinada al autoconsumo y la comercialización. El principal cultivo de la zona es el maíz y el café, seguido de la tagua, maní, cítricos y las verduras para el auto consumo.

El cultivo que ocupa la mayor extensión de una finca es el maíz en asocio con plantas que dan sombra, seguido del café, los que se utilizan para la venta, luego en proporción le siguen el arroz y el resto de cultivos como se observa en la tabla que son destinados para el autoconsumo debido a la dificultad que implica su transportación y su bajo precio en el mercado.

El café se comercializa en la cabecera cantonal a través de los intermediarios que son los que fijan su precio. Las mujeres se encargan de la recolección del café, cosecha de naranja, siembra de maíz y en la cría de animales menores, las otras tareas agrícolas son de responsabilidad exclusiva de los hombres, así como la comercialización agropecuaria. Bautista, C. A. (2004).Tabla \#5.

\section{USO DEL SUELO}

\begin{tabular}{|l|l|l|}
\hline PRODUCTO & $\mathrm{N}^{\circ}$ DE HECTÁREAS & $\%$ \\
\hline Guanábano & 1 & $0,01 \%$ \\
\hline Maracuyá & 2 & $0,03 \%$ \\
\hline Haba & 4 & $0,06 \%$ \\
\hline Guineo & 11 & $0,16 \%$ \\
\hline Plátano & 15 & $0,22 \%$ \\
\hline Maní & 30 & $0,45 \%$ \\
\hline Arroz & 50 & 0,75 \\
\hline
\end{tabular}


IGM-MAGAP-SNI. (Diciembre de 2015).

Productos que cosechan \% por ha.Gráfico \#4

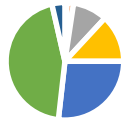

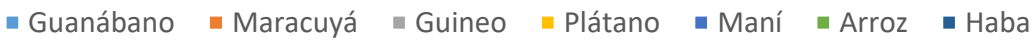

La parroquia rural de Pedro Pablo Gómez, presenta suelos con factores limitantes, profundos, de textura franco arcillosa. Tienen una estructura masiva y son sensibles a la desecación en caso de riegos. Con factores limitantes, pendientes de 50 a 70\%, profundidad débil, éstos son suelos de declives de colinas, pendientes muy fuertes, profundidad variable, pero buena textura; zonas de relieves para pastos o cultivos arbustivos en las zonas húmedas. Tabla \# 6 .

\section{Características de los Suelos.}

\begin{tabular}{|c|c|c|c|c|}
\hline $\begin{array}{l}\text { Características de los } \\
\text { suelos }\end{array}$ & Régimen de humedad & \multicolumn{2}{|c|}{ Taxonomía } & $\begin{array}{l}\text { Material parental y variedad } \\
\text { morfológica }\end{array}$ \\
\hline \multicolumn{5}{|c|}{$\begin{array}{l}\text { Suelos con características "Vertic" más de } 35 \% \text { arcilla tipo motmorillonita, estructura masiva en húmedo grietas de más de } 1 \mathrm{~cm} \text { de ancho en } \\
\text { verano. }\end{array}$} \\
\hline $\begin{array}{l}\text { Grietas cerradas menor } \\
\text { de } 60 \text { días } \mathrm{pH}>7 \mathrm{~B} \text {. I altas }\end{array}$ & Suelos profundos & Aridico & $\begin{array}{l}\text { Vertic camborthid } y / o \\
\text { torrert }\end{array}$ & $\begin{array}{l}\text { Colinas sedimentarias } \\
\mathrm{P}<25 \%\end{array}$ \\
\hline $\begin{array}{l}\text { Grietas abiertas menos de } \\
90 \text { días al año } \mathrm{pH}<7\end{array}$ & $\begin{array}{l}\text { Suelos poco profundos con } \\
\text { CO3Ca en la profundidad } \\
\text { epipedon a veces friable }\end{array}$ & Údico & Paralithic vertic eutropept & $\begin{array}{l}\text { Colinas de lutita blanca P } 25 \text { y } \\
70 \%\end{array}$ \\
\hline \multicolumn{5}{|c|}{ Suelos con horizontes argilico cambio textural abrupto entre el $\mathrm{A} 2$ y el Bt } \\
\hline $\begin{array}{l}\text { Suelos arcillosos con } \\
\text { características "Vertic" }\end{array}$ & $\begin{array}{l}\text { Bt masivo } \\
\text { Sin Co3Ca }\end{array}$ & Ústico & Udertic paleustaef & Colinas sedimentarias $\mathrm{P}<12 \%$ \\
\hline \multicolumn{5}{|c|}{ Suelos con epipedon mólico } \\
\hline \multirow[t]{3}{*}{$\begin{array}{l}\text { Suelos limosos a limo } \\
\text { arenosos poco profundos }\end{array}$} & $\mathrm{Sin} \mathrm{CO} 3 \mathrm{Ca}$ & Údico & Entic hapludoll & $\begin{array}{l}\text { Vertientes de las mesas de } \\
\text { arenisca } \mathrm{P}>70 \%\end{array}$ \\
\hline & Con $\mathrm{CO} 3 \mathrm{Ca} \mathrm{pH}>7$ & Ústico & Entic haplustoll & $\begin{array}{l}\text { Vertientes de las mesas de } \\
\text { arenisca } \mathrm{P}>70 \%\end{array}$ \\
\hline & Poco profundos & Údico & Eratic hapludoll & $\begin{array}{l}\text { Colinas volcano sedimentarias } \\
\mathrm{P} \text { de } 25 \text { a } 40 \%\end{array}$ \\
\hline $\begin{array}{l}\text { Suelos limo - arcillosos a } \\
\text { arcillosos }\end{array}$ & Profundos & Údico & Typic y Vertic hapludoll & $\begin{array}{l}\text { Mesas de arenisca y colinas } \\
\text { volcano sedimentarios P de } 12 \\
\text { a } 40 \%\end{array}$ \\
\hline \multicolumn{2}{|c|}{$\begin{array}{l}\text { Suelos arcillosos en forma general (más } \\
\text { limosos en las cabezas de los valles }\end{array}$} & vertic sutropept & \multicolumn{2}{|c|}{ Valles fluviales indiferenciados } \\
\hline
\end{tabular}

Fuente: IGM-MAGAP-SNI.

\section{Características Químicas de los Suelos. (Tabla \#7)}

\begin{tabular}{|c|c|c|}
\hline Componente & Valores Referenciales & Pedro Pablo Gómez \\
\hline $\mathrm{pH}$ & $6.0-7.5$ & 7,9 \\
\hline $\mathrm{N}$ & $40 \mathrm{ppm}$ & 27,67 \\
\hline $\mathrm{P}$ & $14 \mathrm{ppm}$ & 29,33 \\
\hline $\mathrm{K}$ & $0.38 \mathrm{meq} / 100 \mathrm{ml}$ & 3,17 \\
\hline
\end{tabular}


Raquel Vera Velázquez., Kirenia Maldonado Zúñiga, Wilfrido Javier Del Valle Holguín...

\begin{tabular}{|c|c|c|}
\hline $\mathrm{Ca}$ & $8.9 \mathrm{meq} / 100 \mathrm{ml}$ & 22,67 \\
\hline $\mathrm{Mg}$ & $2.3 \mathrm{meq} / 100 \mathrm{ml}$ & 23 \\
\hline $\mathrm{S}$ & $20 \mathrm{ppm}$ & 0,93 \\
\hline $\mathrm{Zn}$ & $7 \mathrm{ppm}$ & 8,67 \\
\hline $\mathrm{Cu}$ & $4 \mathrm{ppm}$ & 13,67 \\
\hline $\mathrm{Fe}$ & $40 \mathrm{ppm}$ & 5,7 \\
\hline $\mathrm{Mn}$ & $15 \mathrm{ppm}$ & 0,65 \\
\hline $\mathrm{B}$ & $0,40 \mathrm{ppm}$ & 1 \\
\hline $\mathrm{MO}$ & $>5 \%$ & 4,73 \\
\hline $\mathrm{Ca} / \mathrm{Mg}$ & $2.6-8.0$ & 1,58 \\
\hline $\mathrm{Mg} / \mathrm{K}$ & $7.5-15$ & 8,95 \\
\hline $\mathrm{Ca}+\mathrm{Mg} / \mathrm{K}$ & $27.5-55.0$ & Franco Arcilloso \\
\hline $\mathrm{Textura}$ & & \\
\hline
\end{tabular}

Fuente: IGM-MAGAP-SNI.

Identificación de Problemas y Potencialidades del Componente Biofísico. Tabla\#8

\begin{tabular}{|l|l|l|}
\hline \multicolumn{1}{|c|}{ Variable } & \multicolumn{1}{|c|}{ Potencialidades } & \multicolumn{1}{c|}{ Problemas } \\
\hline $\begin{array}{l}\text { Uso y cobertura } \\
\text { del suelo }\end{array}$ & Suelos fértiles y productivos & $\begin{array}{l}\text { Desconocimiento por parte de los } \\
\text { habitantes de las bondades del suelo } \\
\text { fértil y su utilización para la producción } \\
\text { agrícola. }\end{array}$ \\
\hline Agua & $\begin{array}{l}\text { La parroquia posee una } \\
\text { cuenca hídrica que podría } \\
\text { abastecer del líquido vital a } \\
\text { los habitantes de la misma. }\end{array}$ & $\begin{array}{l}\text { Descargas de aguas negras desde la } \\
\text { población de parroquia y comunidades } \\
\text { como las de desagües de alcantarillado }\end{array}$ \\
\hline Biodiversidad & $\begin{array}{l}\text { Existe en la zona montañosa } \\
\text { una fauna diversa. }\end{array}$ & $\begin{array}{l}\text { Presencia de cazadores foráneos que } \\
\text { asechan a las especies que se encuentran } \\
\text { en la zona montañosa, con el fin de } \\
\text { comercializar a estas especies. }\end{array}$ \\
\hline
\end{tabular}

Fuente: IGM-MAGAP-SNI.

RELACION DEL PROYECTO INTEGRADOR DE SABERES CON LAS CIENCIAS BASICAS (G.M.Rs.003-06-2017). Tabla \#9

\begin{tabular}{|l|l|l|l|l|}
\hline Química & Física & Matemáticas & Biología & Expresión Oral \\
\hline $\begin{array}{l}\text { Estructura química del } \\
\text { suelo, pH, salinidad }\end{array}$ & $\begin{array}{l}\text { Estructura física del } \\
\text { suelo }\end{array}$ & $\begin{array}{l}\text { Población agrícola } \\
\text { Población no agrí́cola } \\
\text { Hombres y mujeres } \\
\text { Tablas estadísticas con medidas } \\
\text { de tendencia central(media } \\
\text { moda, mediana y rango) Anexos }\end{array}$ & Cultivos utilizados & $\begin{array}{l}\text { Sistematización de } \\
\text { la información }\end{array}$ \\
\hline $\begin{array}{l}\text { Clasificación de los } \\
\text { suelos por } \\
\text { características } \\
\text { químicas sus }\end{array}$ & $\begin{array}{l}\text { Clasificación de los } \\
\text { suelos por sus } \\
\text { características físicas }\end{array}$ & $\begin{array}{l}\text { Uso del suelo: Superficies } \\
\text { cultivadas por cultivo } \\
\text { Resolver problemas aplicados al } \\
\text { entorno agropecuario }\end{array}$ & $\begin{array}{l}\text { Tipos } \\
\text { microorganismos de } \\
\text { suelo }\end{array}$ & de \\
la información
\end{tabular}




\begin{tabular}{|c|c|c|c|c|}
\hline $\begin{array}{l}\text { Uso de plaguicidas, } \\
\text { para el control de } \\
\text { enfermedades, plagas }\end{array}$ & $\begin{array}{l}\text { Condiciones físicas } \\
\text { del clima } \\
\mathrm{T}^{\circ}, \mathrm{HR} \text {, altura y pp }\end{array}$ & $\begin{array}{l}\text { Coordenadas de la zona latitud, } \\
\text { longitud, altura Resolver } \\
\text { problemas aplicados al entorno } \\
\text { agropecuario }\end{array}$ & Animales que se crían & $\begin{array}{l}\text { Sistematización de } \\
\text { la información }\end{array}$ \\
\hline $\begin{array}{l}\text { Uso de plaguicidas } \\
\text { para el control de } \\
\text { malezas }\end{array}$ & $\begin{array}{l}\text { Disponibilidad de } \\
\text { agua para riego en la } \\
\text { zona }\end{array}$ & $\begin{array}{l}\text { Pérdidas causadas por } \\
\text { enfermedades y plagas en los } \\
\text { cultivos. } \\
\text { Resolver problemas aplicados al } \\
\text { entorno agropecuario }\end{array}$ & $\begin{array}{l}\text { Principales } \\
\text { enfermedades de los } \\
\text { cultivos }\end{array}$ & $\begin{array}{l}\text { Sistematización de } \\
\text { la información }\end{array}$ \\
\hline $\begin{array}{l}\text { Fertilizantes utilizados } \\
\text { en los cultivos }\end{array}$ & $\begin{array}{l}\text { Clasificación de los } \\
\text { suelos según su uso }\end{array}$ & $\begin{array}{l}\text { Cantidad de animales criados por } \\
\text { especie } \\
\text { Resolver problemas aplicados al } \\
\text { entorno agropecuario }\end{array}$ & $\begin{array}{l}\text { Principales plagas de } \\
\text { los cultivos }\end{array}$ & $\begin{array}{l}\text { Sistematización de } \\
\text { la información }\end{array}$ \\
\hline
\end{tabular}

\section{CONCLUSIONES}

Los suelos de la parroquia Pedro Palo Gómez son de textura franco arcilloso ligeramente alcalino. Sin embargo, presentan serias limitantes ya que en su mayoría son suelos con pendientes que sobrepasan los $70 \%$.

Los agricultores de la parroquia Pedro Pablo Gómez frecuentemente aplican agroquímicos en sus cultivos, lo cual conlleva a largo tiempo una degradación de los suelos.

Los agricultores de la parroquia Pedro Pablo Gómez, usan fertilizantes de origen químico en sus cultivos, sin embargo, en gran proporción mencionan que poco a poco han empezado a aplicar humus o biol en sus cultivos lo que demuestra que han mejorado sus prácticas de cultivo.

\section{BIBLIOGRAFÍA}

Bautista, C. A. (2004). La Calidad del Suelo y sus Indicadores. Revista Ecosistemas. Obtenido de http://www.revistaecosistemas.net/articulo.asp?Id=149.

Gómez, G. P. (2015). Plan de Desarrollo y Ordenamiento Territorial. Obtenido de http://app.sni.gob.ec/snilink/sni/PORTAL_SNI/data_sigad_plus/sigadplusdocumentofinal/1360042980001_PDYOT \%20PEDRO\%20PABLO\%20GOMEZ\%20JIPIJAPA_02-07-2016_09-47-12.pdf.

Guía Metodológica Para la Elaboración del Proyecto Integrador de Saberes en la Universidad Estatal del sur de Manabí. Resolución número 003-06-2017.

Intriago, B. R. (Diciembre de 2013). Evaluación del Suelo Bajo Indicadores Agroecológicos de tres Fincas Arroceras del Cantón Daule. Obtenido de Universidad Católica Santiago de Guayaquíl: http://repositorio.ucsg.edu.ec/bitstream/3317/1550/1/T-UCSG-PRE-TEC-AGRO-27.pdf.

MAGAP. (Diciembre de 2015). Memoria técnica geopedológicas de la carta de Azogues. Obtenido de http://www.partealta.ec/opinion/153-editorial/15761.

Parker RO. 2010. Plant and soil science: Fundamentals and applications. Clifton park,

Delmar Cengage Learning, NY. 480 pp.

Senplades (Secretaría Nacional de Planificación y Desarrollo) (2010). Agenda Zonal para el Buen Vivir. Propuestas de Desarrollo y Lineamientos para el Ordenamiento Territorial 2010. Quito: Imprenta Monsalve Moreno.

Tamhane RV, Motiramani DP, YP Bali y DonahueRL. 1986. Suelos: su química y fertilidad en zonas tropicales. Editorial Diana Técnico. México D.F. 483 pp. United States Department of Agriculture. 
Raquel Vera Velázquez., Kirenia Maldonado Zúñiga, Wilfrido Javier Del Valle Holguín... 